\title{
A Widely Tunable Vernier Filter on a Ge-on-SOI Platform for Sensing Applications
}

\author{
Sanja Radosavljevic, Nuria Teigell Beneitez, Andrew Katumba, Muhammad Muneeb, Michael \\ Vanslembrouck, Bart Kuyken and Gunther Roelkens \\ Photonics Research Group, Ghent University - imec, Technologiepark 15, 9052 Ghent \\ Center for Nano- and Biophotonics, Technologiepark 15, 9052 Ghent, Belgium \\ sanja.radosavljevic@ugent.be
}

\begin{abstract}
We present a Vernier tunable racetrack resonator filter on Ge-on-SOI, with $110 \mathrm{~nm}$ FSR at $5 \mu \mathrm{m}$. The racetrack has a Q-factor of 20000 and side-peak suppression $>20 \mathrm{~dB}$, sufficient for wavelength selection in an external cavity laser.

OCIS codes: (130.7408) Wavelength filtering devices; (130.3120) Integrated optics devices; (130.3060) Infrared; (160.6840) Thermo-optical materials; (220.4241) Nanostructure fabrication;
\end{abstract}

\section{Introduction}

The silicon photonics has a wide range of applications, including spectroscopic sensing, which plays an important role in environmental monitoring, chemical process control, security and medical applications [1,2]. Lately, the research is shifting towards mid-infrared due to the orders of magnitude higher optical absorption cross-section in this range as compared to the telecom and visible wavelength range [3]. Germanium-on-Silicon-on-Insulator (Geon-SOI) emerged as a promising solution for mid-infrared sensing platform in wavelength range 4-12 $\mu \mathrm{m}[4,5]$.

Currently available tuning mechanisms for broadband gain mid-infrared lasers hinder their application in sensing, as wavelength tuning often relies on an external diffraction grating. An interesting implementation of widely tunable mid-IR lasers on this Ge-on-SOI platform is to butt couple a midIR III-V gain chip to the silicon photonic integrated circuit (PIC) that comprises a widely thermally tunable Vernier filter for the laser wavelength selection (Figure $1 \mathrm{a}$ ). One of gain materials of interest in the mid-infrared are quantum cascade (QC) structures, which have already been demonstrated with watt-level output power operating at room temperature in the midinfrared region [6]. A PIC design that can be used to test the Vernier functionality on Ge-on-SOI platform is shown in Figure $1 \mathrm{~b}$ ).
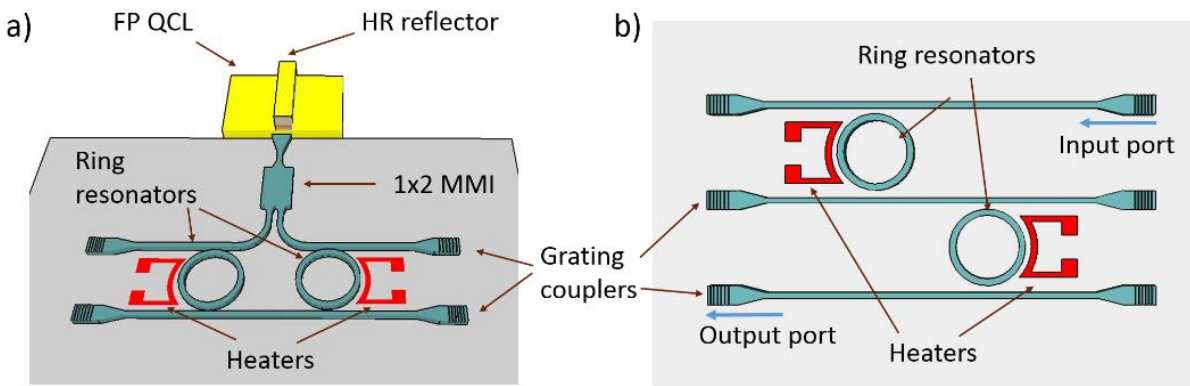

Figure 1 A widely tunable external cavity QCL without any moving parts: the rotating grating is replaced by a tunable reflector on a chip. The tunable filter consists of a beam combiner/splitter and a Vernier ring resonator filter in a loop configuration (a), a tunable Vernier filter based on ring resonators $(b)$.

\section{Simulation Results}

The free spectral range (FSR) of the Vernier filter FSR , realized by cascading the individual resonators, depends on the individual FSRs of the racetrack resonators, $\mathrm{FSR}_{1}$ and $\mathrm{FSR}_{2}$, and reads:

$$
\mathrm{FSR}_{\mathrm{v}}=\left|\frac{\mathrm{FSR}_{1} \mathrm{FSR}_{2}}{\mathrm{FSR}_{2}-\mathrm{FSR}_{1}}\right|
$$

In our design, $\mathrm{FSR}_{1}$ and $\mathrm{FSR}_{2}$ are $9.3 \mathrm{~nm}$ and $8.6 \mathrm{~nm}$ respectively in the $5 \mu \mathrm{m}$ wavelength range, leading to a combined FSR $\mathrm{F}_{\mathrm{V}}$ of $114 \mathrm{~nm}$ and the side-peak suppression of $>15 \mathrm{~dB}$. The transmission of the Vernier filter is shown in Figure 2 a). 

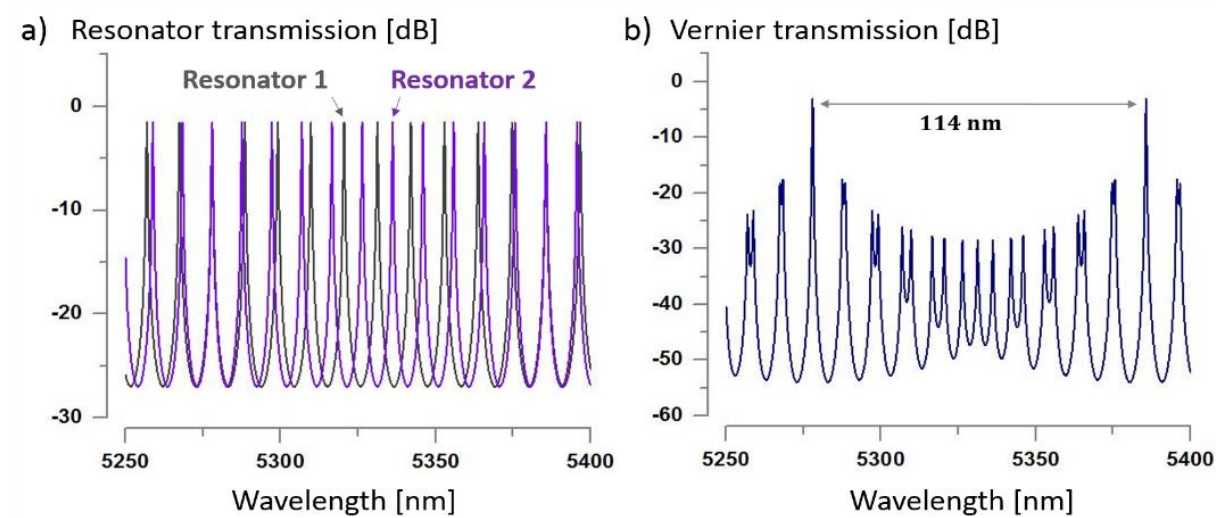

Figure 2 The simulated transmission spectra of individual racetrack resonators (a) and the transmission of the Vernier filter built with these racetracks $(b)$.

\section{Measurement results}

The fabricated racetrack resonators have $\mathrm{Q}$ factor around 20000. By thermally tuning one of the racetrack resonators the position of the overlapping transmission peak can be tuned in steps of the FSR of the other resonator. This way, discrete tuning of the filter can be realized as shown in Figure 3 a). The heater power used for resonator $1, \mathrm{P}_{1}$, and that for resonator 2, $\mathrm{P}_{2}$, indicated as $\left(\mathrm{P}_{1}, \mathrm{P}_{2}\right)$. To tune through the $\mathrm{FSR}_{\mathrm{V}}$ of the filter requires $250 \mathrm{~mW}$ power dissipation. The side-peak suppression of the filter transmission is more than $20 \mathrm{~dB}$. In order to access wavelengths in between resonances of the resonator that was not tuned, both racetrack resonators need to be tuned at the same time. This is illustrated in Figure 3 b).

a)

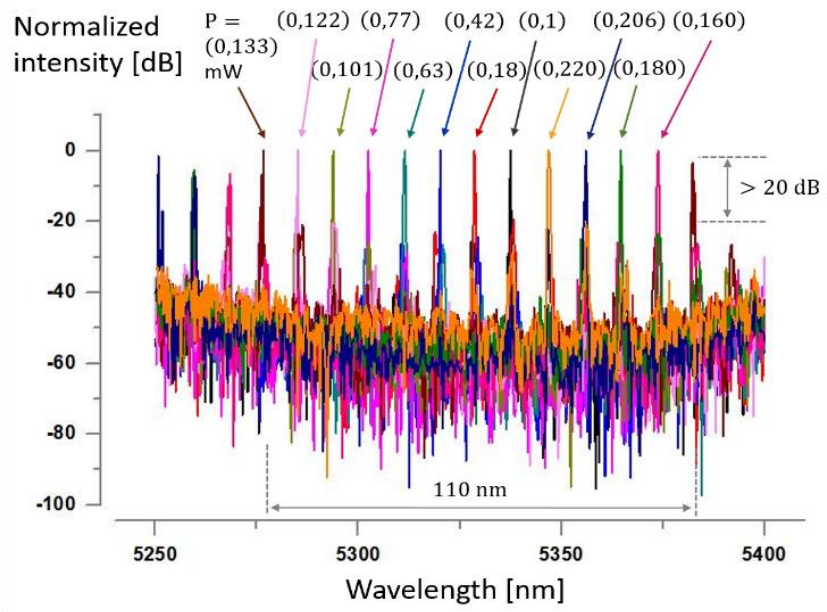

b)

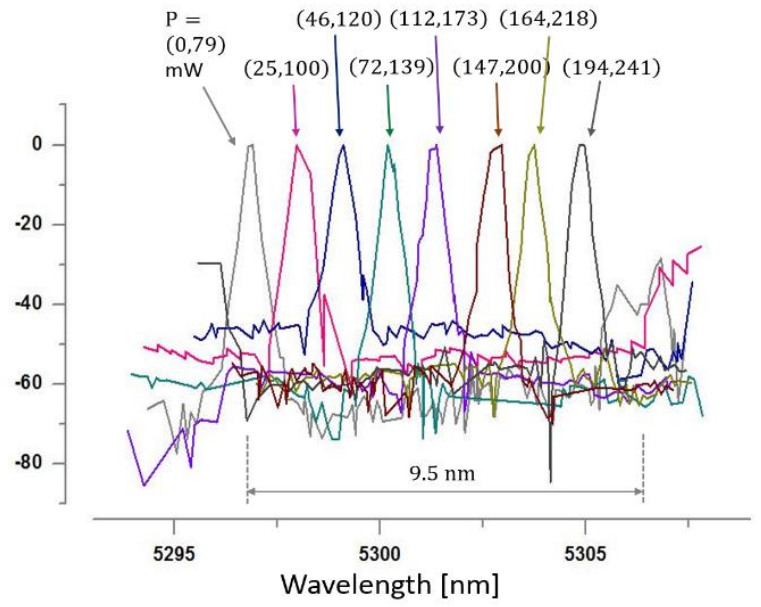

Figure 3 The measured normalized intensity spectra of discrete Vernier tuning (a) and the normalized intensity spectra of the continuous tuning of the same Vernier filter $(b)$.

\section{References}

[1] J. T. Robinson, C. Long, and M. Lipson, “On-chip gas detection in silicon optical microcavities," Opt. Express 16, 4296-4301 (2008).

[2] F. Cunin, T. A. Schmedake, J. R. Link, Y. Y. Li, J. Koh, S. N. Bhatia, and M. J. Sailor, "Biomolecular screening with encoded porous-silicon photonic crystals," Nature materials 1, 39-41 (2002).

[3] P. A. Werle, "Review of recent advances in semiconductor laser based gas monitors," Spectrochim. Acta Mol. Biomol. Spectrosc. 54, 197236 (1998).

[4] A. Malik, S. Dwivedi, L. Van Landschoot, M. Muneeb, Y. Shimura, G. Lepage, J. Van Campenhout, W. Vanherle, T. Van Opstal, R. Loo, and G. Roelkens, "Ge-on-Si and Ge-on-SOI thermo-optic phase shifters for the mid-infrared," Opt. Express 22, 28479-28488 (2014).

[5] S. Radosavljevic, B. Kuyken, and G. Roelkens, "Efficient $5.2 \mu \mathrm{m}$ wavelength fiber-to-chip grating couplers for the Ge-on-Si and Ge-on-SOI mid-infrared waveguide platform," Opt. Express 25, 19034-19042 (2017).

[6] Q. Y. Lu, Y. Bai, N. Bandyopadhyay, S. Slivken, and M. Razeghi, "Room-temperature continuous wave operation of distributed feedback quantum cascade lasers with watt-level power output,” App. Phys. Lett. 97, 231119 (2010). 